\section{Isabelle Bairati}

\section{ADRESSE}

I. Bairati : professeur. Département de médecine sociale et préventive, groupe de recherche en épidémiologie, faculté de médecine université Laval, Québec, QC, G1K 7P4, Canada.

\section{TIRÉS A PART}

I. Bairati.

\title{
Effets de l'huile de poisson sur la resténose coronarienne après angioplastie
}

L'angioplastie coronaire transluminale par voie percutanée est aujourd'hui une technique de choix dans le traitement des sténoses coronaires. Son intérêt est cependant limité par la fréquence des resténoses dans les six mois qui suivent l'acte thérapeutique (30\% à $45 \%)$. Les huiles de poisson, riches en acides gras $\omega 3$, pourraient avoir une action sur les différentes étapes conduisant à la resténose. Elles ont fait l'objet de huit essais thérapeutiques dont les résultats sont contradictoires. En fait, la resténose n'a été correctement évaluée, par angiographie et chez tous les patients, que dans quatre de ces études, et, parmi celles-ci, deux seulement s'appuient sur un examen quantitatif informatisé des angiographies. La mise en évidence, dans ces deux études, d'un effet inhibiteur des huiles de poisson sur la resténose est donc particulièrement intéressante. De plus, l'une de ces études montre que des apports alimentaires en acides gras $\omega 3$ supérieurs à $0,15 \mathrm{~g}$ par jour abaissent de façon significative la fréquence des resténoses. L'effet de régimes alimentaires riches en produits de la mer mériterait, par conséquent, d'être plus amplement étudié.

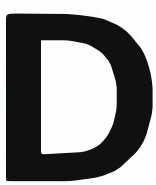

epuis son introduction en 1977 par Andreas R. Gruentzig [1], l'angioplastie coronaire percutanée est devenue une technique de choix dans le traitement de la sténose coronaire. En 1987, la Commission on Professional and Hospital Activities aux États-Unis évaluait à 175000 le nombre de patients ayant bénéficié d'une angioplastie coronaire [2]. Les indications de l'angioplastie n'ont cessé de s'élargir au cours des années. Comparés aux patients traités entre 1977 et 1981, ceux traités entre 1985 et 1986 étaient plus âgés, avec un pronostic cardiaque plus défavorable [3]. L'angioplastie est aussi devenue l'un des traitements de la maladie coronaire multifocale. La technique n'a cessé de s'améliorer, faisant passer la proportion de succès initiaux aux États-Unis de $67 \%$ entre 1977 et 1981, à $88 \%$ entre 1985 et 1986 [3]. Cependant, l'intérêt à moyen terme de l'angioplastie coronaire reste limité par la fréquence de la récidive de la sténose 
1. Gruentzig AR. Transluminal dilatation of coronary artery stenosis. Lancet 1978 ; 1 : 263.

2. Cox JL, Gotlieb Al. Review of antiplatelet drug use in preventing restenosis following percutaneous transluminal coronary angioplasty. Can J Cardiol 1988 ; 4 : 201-9.

3. Detre K, Holubkov R, Kelsey S, et al. Percutaneous transluminal coronary angioplasty in 1985-1986 and 1977-1981: The National Heart, Lung, and Blood Institute Registry. N Engl J Med 1988 ; 318 : 265-70.

4. El-Tamini H, Davies G, Hackett D, Fragasso $G$, Crea $F$, Maseri $A$. Very early prediction of restenosis after successful coronary angioplasty : anatomic and functional assess ment. J Am Coll Cardiol 1990 ; 15 : 259-64

5. Macdonald RG, Henderson MA, Hirshfeld JW, et al. Patient-related variables and restenosis after percutaneous transluminal coronary angioplasty. A report from the $\mathrm{M}$ Heart group. $A m J$ Cardiol 1990 ; 66 : 926-31.

6. Nobuyoshi M, Kimura T, Nosaka H, et al. Restenosis after successful percutaneous transluminal coronary angioplasty : serial angiographic follow-up of 229 patients. $J \mathrm{Am}$ Coll Cardiol 1988; 12 : 616-23.

7. Slack JD, Pinkerton CA, VanTassel J, Orr CM, Scott M, Allen B, Nasser W. Can oral fish oil supplement minimize re-stenosis after percutaneous transluminal coronary angioplasty? J Am Coll Cardiol 1987 ; 9 : $64 \mathrm{~A}$.

8. Dehmer GJ, Popma JJ, van den Berg $\mathrm{EK}$, et al. Reduction in the rate of carly restenosis after coronary angioplasty by a diet supplemented with $\omega 3$ fatty acids. $N$ Engl $J$ Med 1988 ; 319 : 733-40.

9. Grigg LE, Kay TWH, Valentine PA. Determinants of restenosis and lack of effect of dietary supplementation with cicosapentaenoic acid on the incidence of coronary artery restenosis after angioplasty. $\mathrm{J} \mathrm{Am} \mathrm{Coll}$ Cardiol 1989; 13 : 665-72.

10. Milner MR, Gallino RA, Leffingwell $A$, et al. Usefulness of fish oil supplements in preventing clinical evidence of restenosis after percutaneous transluminal coronary angioplasty. Am J Cardiol 1989 ; 64 : 294-9.

11. Reis GJ, Boucher TM, Sipperly ME, vention of restenosis after coronary angioplasty. Lancet $1989 ; 2$ : 177-81.

12. Nye ER, llsley CD, Ablett MB, Sutherland WHF, Robertson MC. Effect of eicosapentaenoic acid on restenosis rate, clinical course and blood lipids in patients after percutaneous transluminal coronary angioplasty. Aust NZ J Med 1990 ; 20 : 549-52.

13. Kaul U, Sanghvi S, Bahl VK, Dev V, Wasir HS. Fish oil supplements for prevention of restenosis after coronary angioplasty. Int J Cardiol 1992; 35 : 87-93

14. Bairati I, Roy L, Meyer F. Doubleblind randomized controlled trial of fish oil supplements in prevention of recurrence of stenosis after coronary angioplasty. Circulation $1992 ; 85: 950-6$.

15. Dyerberg J, Bang HO, Hjorne N. Fatty acid composition of the plasma lipids in Greenland Eskimos. Am J Clin Nutr 1975 ; 28 : 958-66.

16. Leaf A. Cardiovascular effects of fish oil : beyond the platclet. Circulation 1990 ; 82 : 624-8.

17. Kinsella JE, Lokesh B, Stone RA. Dietary $\omega 3$ polyunsatured fatty acids and amelioration of cardiovascular disease : possible mechanisms. Am J Clin Nutr 1990; 52 : $1-28$.

18. Lee TH, Hoover RL, Williams JD, et al. Effect of dietary enrichment with eicosapentaenoic and docosahexaenoic acids on in vitro neutrophil and monocyte leukotriene generation and neutrophil function. $N$ Engl J Med 1985 ; 312 : 1217-24.

19. Shimokawa H, Lam JYT, Chesebro JH, Bowie EJW, Vanhoutte PM. Effects of dietary supplementation with cod-liver oil on endothelium-dependent responses in porcine coronary arteries. Circulation 1987; 4: 898-905.

20. Stein B, Fuster V, Chesebro JH. Drugs and coronary angioplasty. In : Meier B, ed. Interventional cardiology. Toronto : Hogrefe and Huber Publishers, 1990: 11-30.

21. Siegel RJ, Chae JS, Forrester JS, Ruiz CE. Angiography, angioscopy, and ultrasound imaging before and after percutaneous balloon angioplasty. Am Heart J $1990 ; 120$ : 1086-90. (ou resténose) au site de la dilatation. Six mois après angioplastie, le pourcentage de resténose varie entre $30 \%$ et $45 \%[4-6]$. Aucun traitement médicamenteux (aspirine, corticostéroïdes, antagonistes du calcium) n'a démontré à ce jour son efficacité dans la prévention de la resténose après angioplastie [2]. En revanche, les suppléments d'huile de poisson, riches en acides gras polyinsaturés $\omega 3$, semblent apporter des résultats prometteurs dans cette prévention [7-14].

\section{Mécanismes d'action de I'huile de poisson}

Les huiles de poisson sont riches en acides gras polyinsaturés $\omega 3$ à longue chaîne carbonée, en particulier en acide eicosapentaénoïque (EPA) et docosahexaénoïque (DHA). En 1975, Dyerberg et al. [15] notaient une plus grande proportion d'EPA dans les lipides sanguins des Inuit vivant au Groenland que dans ceux des Danois. Les auteurs émirent alors l'hypothèse que la faible incidence des cardiopathies ischémiques observées dans cette population pouvait être attribuée à une alimentation riche en EPA.

Depuis les observations de Dyerberg et al., diverses expérimentations ont permis de connaître les mécanismes d'action des acides gras polyinsaturés $\omega 3$ sur les facteurs de risque de la maladie coronaire. L'EPA et le DHA entrent en compétition avec l'acide arachidonique et modifient la synthèse des endoperoxydes $[16,17]$. Dans les plaquettes, on observe une diminution de la synthèse de thromboxane A2 et l'apparition de faibles quantités physiologiquement inactives de thromboxane A3. Dans les cellules endothéliales, des prostaglandines 3 sont synthétisées. Ces modifications métaboliques entraînent une inhibition de l'agrégation plaquettaire et une vasodilatation $[16,17]$. Les huiles de poisson ont également une action anti-inflammatoire, en agissant sur la chimiotaxie et l'agrégation des neutrophiles [18]. Les huiles de poisson pourraient aussi diminuer la vasoconstriction en réponse aux catécholamines et à l'angiotensine, ainsi que le niveau de fibrinogène et inhiber l'hyperplasie intimale [16, 19, 20]. 


\section{Mécanismes d'action de I'angioplastie}

Les mécanismes thérapcutiques de l'angioplastie sont encore mal connus ; trois mécanismes sont cependant fréquemment évoqués. Le ballonnet, introduit dans l'artère coronaire et gonflé en regard de la lésion, entraîne des dommages de la paroi artérielle. La plaque d'athérome est alors fissurée, créant des tunnels dans lesquels le sang peut circuler [20]. Une dissection peut se produire à travers l'intima jusqu'à la média, créant un élargissement du vaisseau [20, 21]. Dans les lésions excentriques, le ballonnet peut dilater la paroi artérielle dépourvue de plaque d'athérome et conduire à une augmentation du diamètre du vaisseau [20-22].

\section{Mécanisme de formation de la resténose}

L'action mécanique de l'angioplastie induit l'activation de divers facteurs biologiques. Les plaquettes viennent rapidement adhérer au niveau du vaisseau endommagé, et les substances agrégantes, comme l'adénosine diphosphate et la thromboxane A2, sont libérées [20]. L'activation des plaquettes, ainsi que celle des macrophages, des cellules endothéliales et des cellules musculaires lisses, entraîne la libération de facteurs mitogènes. La prolifération cellulaire, en particulicr celle des cellules musculaires lisses, serait à l'origine de la resténose [20, 23]. De surcroît, la production de thromboxane A2 par les plaquettes activées entraînerait une vasoconstriction au niveau de la lésion, ajoutant un facteur spastique au phénomène de resténose [22]. Un autre processus mécanique de la resténose pourrait intervenir, avec l'incorporation dans la paroi du vaisseau d'un thrombus plaquettaire organisé [20].

\section{Évaluation de la resténose}

Le degré de sténose avant et après angioplastie est évalué à partir des images angiographiques. De même, la resténose est habituellement évaluée sur les angiographies de contrôle, effectuées plusieurs mois après la dilatation. Le diagnostic angiographique de resténose peut reposer sur plu$\mathrm{m} / \mathrm{s} n^{\circ} 10 \mathrm{vol}$. 9, octobre 93 sicurs définitions de la resténose et sur différentes méthodes de lecture des angiographics. Trois définitions de resténose sont le plus couramment utilisées : (1) sur la base de concepts physiologiques et d'expérimentations animales, la resténose est définie par un diamètre final, au niveau de la sténose, inférieur à $50 \%$ du diamètre du vaisseau sain [23] ; (2) la définition proposée par le National Heart, Lung, and Blood Institute, et fréquemment utilisée en pratique hospitalière, considère qu'il y a resténose si au moins la moitié du gain obtenu par l'angioplastie dans le diamètre du vaisseau est perdue à la fin du suivi [24] ; (3) la dernière définition a été proposée par Serruys et al. [23], après l'introduction de méthodes quantitatives dans l'évaluation des angiographies : on dit qu'il y a resténose quand le diamètre minimal de la lumière du vaisseau, en fin d'étude, est inférieur au diamètre minimal après dilatation moins deux écarts-types de la différence entre deux mesures du diamètre minimal faites sur la même image. Toutes ces définitions de resténoses sousentendent que, préalablement, l'angioplastie a été un succès. Enfin, le succès est le plus couramment défini par une réduction initiale de la sténose du vaisseau d'au moins
$20 \%$, une sténose après angioplastie de moins de $50 \%$, et l'absence de complications graves durant, ou immédiatement après, l'angioplastie [25].

Deux méthodes de lecture des angiographies sont fréquemment utilisées. Une méthode visuelle, où l'évaluateur compare, avec ou sans l'aide d'un compas, le diamètre de la section non opacifiée par le liquide de contraste au diamètre du vaisseau normal à proximité de la sténose. Depuis l'introduction des ordinateurs, la lecture des angiographies peut être réalisée par méthode quantitative informatisée [26]. Le contour du vaisseau est déterminé automatiquement à partir du contraste produit par l'opacification des vaisseaux coronaires. Le diamètre minimal de la sténose est mesuré automatiquement et comparé au diamètre du vaisseau normal. Cette méthode a l'avantage de donner les diamètres de la sténose et du vaisseau normal en valeur absolue $(\mathrm{mm})$, et de limiter l'intervention des évaluateurs dans la détermination du diamètre de la sténose.

\section{Prévention de la resténose}

Huit essais cliniques randomisés (Tableau I) ont évalué l'efficacité d'un supplément d'huile de poisson riche

\begin{tabular}{|c|c|c|c|}
\hline & $\begin{array}{l}\text { Tableau I } \\
\text { HODES D'ÉVALUATION DE LA REST } \\
\text { ET EFFET DE L'HUILE DE POISSO } \\
\text { UR LA RESTÉNOSE SELON LES ÉTU }\end{array}$ & $\begin{array}{l}\text { NOSE } \\
\text { ES }\end{array}$ & \\
\hline Études & Évaluation de la resténose & Effet & protecteur \\
\hline $\begin{array}{l}\text { Slack et al. } \\
\text { [7] }\end{array}$ & Epreuve d'effort & & oui* \\
\hline $\begin{array}{l}\text { Dehmer et al. } \\
\text { [8] }\end{array}$ & Angiographie & & oui \\
\hline $\begin{array}{l}\text { Grigg et al. } \\
\text { [9] }\end{array}$ & Angiographie & & non \\
\hline $\begin{array}{l}\text { Milner et al. } \\
{[10]}\end{array}$ & Épreuve d'effort ou angiographie & & oui \\
\hline $\begin{array}{l}\text { Reis et al. } \\
{[11]}\end{array}$ & Épreuve d'effort ou angiographie & & non \\
\hline $\begin{array}{l}\text { Nye et al. } \\
{[12]}\end{array}$ & Angiographie & & oui \\
\hline $\begin{array}{l}\text { Kaul et al. } \\
{[13]}\end{array}$ & Épreuve d'effort et/ou angiographie & & non \\
\hline $\begin{array}{l}\text { Bairati et al. } \\
\text { [14] }\end{array}$ & Angiographie & & oui \\
\hline
\end{tabular}

* Effet protecteur chez les patients n'ayant subi qu'une seule dilatation. 


\section{RÉFÉRENCES}

22. Fischell TA, Grant G, Johnson DE. Determinants of smooth muscle injury during balloon angioplasty. Circulation 1990 ; 82 : $2170-84$.

23. Serruys PW, Rensing BJ, Luijten HE, Hermans WR, Beatt KJ. Restenosis following coronary angioplasty. In : Meier B, ed. Interventional cardiology. Toronto : Hogrefe and Huber Publishers, 1990: 79-115.

24. Holmes DR, Vlietstra RE, Smith HC, et al. Restenosis after percutaneous transluminal coronary angioplasty (PTCA) : a report from the PTCA registry of the National Heart, Lung, and Blood Institute. $A m$ J Cardiol 1984; 53 : 77C-81.

25. Bourassa MG, Alderman EL, Bertrand $\mathrm{M}$, et al. Report of the joint ISFC/WHO task force on coronary angioplasty. Special report. Circulation 1988 ; 78 : 780-9.

26. Mancini GBJ, Simon SB, McGillem MJ, LeFree MT, Friedman HZ, Vogel RA. Automated quantitative coronary arteriography : morphologic and physiologic validation in vivo of a rapid digital angiographic method. Circulation 1987 ; 75 : 452-60

27. de Feyter PJ, Serruys PW, van den Brand $\mathrm{M}$, et al. Emergency coronary angioplasty in refractory unstable angina. $N$ Engl $J$ Med 1985 ; 313 : 342-6.

28. Sicss W, Roth P, Scherer B, Kurzmann I, Bohlig B, Weber PC. Platelet membrane fatty acids, platelet aggregation, and thromboxane formation during a mackerel diet. Lancet 1980 ; 1 : 441-4

29. Knapp HR, Reilly IAG, Alessandrini $P$, FitzGerald GA. In vivo indexes of platelet and vascular function during fish-oil administration in patients with atherosclerosis. N Engl J Med 1986 ; 314 : 937-42. en acides polyinsaturés $\omega 3$ dans la prévention de la resténose après angioplastie [7-14]. Cinq études [7, 8, $10,12,14]$ rapportent un effet protecteur de l'huile de poisson, dont l'une ne décrit un effet bénéfique que chez les sujets ayant subi une seule dilatation [7]. Une interprétation globale des résultats de ces huit essais cliniques est difficile à réaliser à cause de la diversité des protocoles d'étude. Les caractéristiques des populations évaluées, le dosage en EPA et DHA des suppléments d'huile de poisson, la durée de la supplémentation avant et après angioplastie, la prise d'autres produits inhibant l'agrégation plaquettaire, diffèrent selon les études et pourraient, au moins en partie, expliquer la divergence des résultats.

Le protocole des huit essais cliniques est important à considérer lors de l'interprétation des résultats, pour estimer le poids à accorder aux résultats de chaque étude. Dans seulement la moitié des essais, le groupe contrôle a reçu un placebo $[9,11,12,14]$, et l'essai n'est réalisé totalement en double aveugle que dans trois études [9, 12, 14]. Dans plusieurs études, les méthodes diagnostiques de la resténose sont critiquables. Slack et al. [7] ont recours à l'épreuve d'effort pour évaluer la récidive de la lésion. Cette méthode apparaît peu appropriée, car non spécifique de la resténose, particulièrement dans une étude où $30 \%$ des sujets présentent des lésions coronaires multiples. Les études de Milner et al. [10], de Reis et al. [11], et de Kaul et al. [13] décrivent une évaluation en deux étapes. La première évaluation permet de classer les patients en symptomatiques et non symptomatiques. L'investigation des sujets symptomatiques comporte soit une angiographie, soit une épreuve d'effort, soit les deux, alors que celle des patients cliniquement asymptomatiques se résume à une épreuve d'effort. Ce type d'évaluation apparaît peu fiable car $11 \%$ à $33 \%$ des sujets sans signes cliniques coronaires, ou avec une épreuve d'effort négative, présentent une resténose à l'angiographie $[9,24,27]$. Compte tenu de l'évaluation angiographique de la lésion initiale et de son aspect résiduel après angioplastie, le diagnostic de resténose devrait toujours être porté sur une angiographie de contrôle.
Quatre essais cliniques sur huit ont évalué la récidive de la sténose à partir d'angiographies de contrôle, mais les méthodes d'évaluation et les définitions de la resténose ne sont pas toujours identiques $[8,9,12,14]$. Trois études rapportent un effet protecteur de l'huile de poisson sur la resténose $[8,12,14]$. Dans la quatrième étude, Grigg et al. [9] observent des pourcentages de resténose similaires chez les patients recevant de l'huile de poisson et chez ceux prenant un mélange d'huile d'olive et d'huile de maïs. Le pourcentage de resténose, évalué visuellement et selon deux définitions de resténose, est d'environ $32 \%$. Ce résultat négatif pourrait s'expliquer en partie par les petites doses d'EPA et de DHA ingérées (3 g/jour) et par la faible durée du suivi (3,5 mois). De plus, l'expérimentation ne prévoyait pas de période de supplémentation avant l'angioplastie, alors que la modification en acides gras des lipides membranaires n'apparaît qu'après une semaine, au moins, d'un régime riche en acides gras $\omega 3[28,29]$. Utilisant l'évaluation visuelle des angiographies et la combinaison de deux définitions de resténose, Nye et al. [12] rapportent un effet favorable de l'huile de poisson sur la resténose. Cependant, le pourcentage de resténose dans le groupe expérimental et le groupe contrôle (huile d'olive) est comparé, non pas par individu, mais par lésion dilatée. Une telle analyse n'est pas adéquate pour des événements non indépendants. En effet, pour un même individu, la probabilité de resténoser une deuxième lésion semble liée au devenir de la première lésion $[14,25]$. Dans le cas où l'huile de poisson aurait un effet favorable sur la resténose, l'association serait alors surestimée. L'étude de Dehmer et al. [8] est un essai randomisé en double aveugle, non contrôlé, dans lequel seule la lecture des angiographies est réalisée en aveugle du traitement. Dans cette étude, la récidive de la sténose est évaluée selon une seule définition de resténose, mais la lecture visuelle des angiographies, pratiquée à l'aide d'un compas, a été validée par une lecture quantitative informatisée. Le pourcentage de resténose dans le groupe traité est de $19 \%$ et de $46 \%$ dans le groupe de 
comparaison (sans placebo). Les doses d'EPA et de DHA sont de $5,4 \mathrm{~g}$ par jour, et la supplémentation a débuté une semaine avant l'angioplastie. Cependant, si la resténose est évaluée par angiographie à trois mois pour l'ensemble des sujets, à six mois elle ne l'est que pour les patients symptomatiques. L'étude de Bairati et al. [14] est un essai clinique contrôlé randomisé, en double aveugle, dans lequel 205 patients, traités pour la première fois par angioplastie, ont reçu $15 \mathrm{~g}$ par jour d'huile de poisson (4,5 g par jour d'EPA et de DHA) ou d'huile d'olive. Le traitement a débuté trois semaines avant l'angioplastie, et s'est poursuivi pendant six mois. En fin d'essai, tous les patients chez lesquels la dilatation avait été réussie ont eu une angiographie de contrôle. Les lésions ont été évaluées par une méthode informatisée quantitative et la resténose a été évaluée selon les quatre définitions angiographiques rapportées plus haut. La resténose est survenue moins souvent dans le groupe traité à l'huile de poisson $(22,0 \%$ à $35,6 \%)$ que dans le groupe contrôle $(40,0 \%$ à $53,3 \%$ ). Cette différence, statistiquement significative pour trois des quatre définitions de resténose, l'est demeurée après ajustement pour d'autres facteurs de risque $(p=0,03)$. Un apport alimentaire en acides gras $\omega 3$ supérieur à $0,15 \mathrm{~g}$ par jour a aussi été associé à une fréquence moindre de resténose, après ajustement pour le traitement et les déterminants de la resténose $(p \leqslant 0,03)$.

\section{Conclusion}

Seuls quatre essais cliniques sur huit recherchent la resténose par angiographie, chez tous les patients, six mois après angioplastie. Deux de ces essais, qui rapportent un effet protecteur de l'huile de poisson sur la resténose, utilisent une méthode de lecture objective et reproductible des angiographies. Dans la première étude [8], l'évaluation visuelle de la resténose, pratiquée à l'aide d'un compas, a été validée par la lecture informatisée quantitative des angiographies. Quant à la deuxième étude [14], en plus d'évaluer toutes les angiographies par une méthode informatisée quantita- tive, elle analyse les résultats selon quatre définitions angiographiques de resténose. Afin de confirmer les résultats positifs de ces deux études, on attend avec intérêt le résultat d'essais contrôlés, randomisés, en double aveugle, incluant un nombre approprié de patients et reposant sur une évaluation adéquate de la resténose (angiographie avec lecture fiable).

Comme le rapporte un des essais cliniques [14], un régime alimentaire riche en produits de la mer semble avoir un effet protecteur contre la récidive de la sténose coronaire six mois après angioplastie. Cet axe de recherche mériterait qu'on lui accorde une plus grande importance et devrait faire l'objet de plus amples investigations

\section{Summary}

Effects of fish oil on coronary restenosis after angioplasty

Percutaneous transluminal coronary angioplasty (PTCA) is an established alternative treatment for patients with coronary artery disease. However, this technic is restricted by complications such as the recurrence of stenosis, or restenosis, in about $30 \%$ to $45 \%$ of the patients, within six months after PTCA. Fish oils, rich in $\omega 3$ fatty acids, may influence different steps in the process of re-stenosis. The effect of fish oil on preventing re-stenosis after PTCA has been evaluated in eight clinical trials that led to conflicting results. In only four studies was the recurrence of stenosis correctly tested, using angiograms in all patients and, among these, only two based their evaluation on quantitative computer analysis. Thus, the positive results of these two studies must be considered with particular attention. In addition, in one of these studies, a dietary intake of $\omega 3$ fatty acids at a dose greater than $0.15 \mathrm{~g}$ per day was associated with a lower frequency of restenosis $(p \leqslant 0.03)$. Further investigations are required to document the effect of a diet rich in $\omega 3$ fatty acids in the prevention of restenosis after PTCA. 